\title{
Efficacy of virtual block objects in reducing the lung dose in helical tomotherapy planning for cervical oesophageal cancer: a planning study
}

Makoto Ito ${ }^{1,2^{*}}$ (D) Hidetoshi Shimizu ${ }^{1}$, Takahiro Aoyama ${ }^{1}$, Hiroyuki Tachibana ${ }^{1}$, Natsuo Tomita ${ }^{1}$, Chiyoko Makita ${ }^{1}$ Yutaro Koide ${ }^{1}$, Daiki Kato ${ }^{1}$, Tsuneo Ishiguchi ${ }^{2}$ and Takeshi Kodaira ${ }^{1}$

\begin{abstract}
Background: Intensity-modulated radiotherapy is useful for cervical oesophageal carcinoma (CEC); however, increasing low-dose exposure to the lung may lead to radiation pneumonitis. Nevertheless, an irradiation technique that avoids the lungs has never been examined due to the high difficulty of dose optimization. In this study, we examined the efficacy of helical tomotherapy that can restrict beamlets passing virtual blocks during dose optimization computing (block plan) in reducing the lung dose.

Methods: Fifteen patients with CEC were analysed. The primary/nodal lesion and prophylactic nodal region with adequate margins were defined as the planning target volume (PTV)-60 Gy and PTV-48 Gy, respectively. Nineteen plans per patient were made and compared (total: 285 plans), including non-block and block plans with several shapes and sizes.

Results: The most appropriate block model was semi-circular, $8 \mathrm{~cm}$ outside of the tracheal bifurcation, with a significantly lower lung dose compared to that of non-block plans; the mean lung volumes receiving $5 \mathrm{~Gy}$, $10 \mathrm{~Gy}$, $20 \mathrm{~Gy}$, and the mean lung dose were $31.3 \%$ vs. $48.0 \%$ ( $p<0.001$ ), $22.4 \%$ vs. $39.4 \%$ ( $p<0.001$ ), $13.2 \%$ vs. $16.0 \%$ $(p=0.028)$, and 7.1 Gy vs. 9.6 Gy $(p<0.001)$, respectively. Both the block and non-block plans were comparable in terms of the homogeneity and conformity indexes of PTV-60 Gy: 0.05 vs. $0.04(p=0.100)$ and 0.82 vs. 0.85 ( $p=0.616)$ , respectively. The maximum dose of the spinal cord planning risk volume increased slightly (49.4 Gy vs. 47.9 Gy, $p=0.002$ ). There was no significant difference in the mean doses to the heart and the thyroid gland. Prolongation of the delivery time was less than $1 \mathrm{~min}(5.6 \mathrm{~min}$ vs. $4.9 \mathrm{~min}, p=0.010$ ).
\end{abstract}

Conclusions: The block plan for CEC could significantly reduce the lung dose, with acceptable increment in the spinal dose and a slightly prolonged delivery time.

Keywords: Cervical oesophageal cancer, Helical tomotherapy, Intensity-modulated radiation therapy, Lung dose, Complete block, Directional block

\footnotetext{
* Correspondence: itou.makoto.292@mail.aichi-med-u.ac.jp

${ }^{1}$ Department of Radiation Oncology, Aichi Cancer Center Hospital, Chikusa-ku, Nagoya, Japan

${ }^{2}$ Department of Radiology, Aichi Medical University Hospital,

Yazako-Karimata, Nagakute, Aichi 480-1195, Japan
} 


\section{Background}

Cervical oesophageal cancer (CEC) is relatively uncommon, representing $4.4 \%$ of all oesophageal cancers [1]. Although surgery has been the primary treatment for CEC, advanced stage is a contraindication for definitive surgery. Additionally, chemoradiotherapy (CRT) is the standard treatment in patients who refuse pharyngolaryngo-oesophagectomy in order to preserve laryngeal function. A prospective study of CEC treated with 3dimensional conformal radiotherapy (3DCRT) reported that CRT provides comparable survival to that of surgical resection [2]. Recently, intensity-modulated radiation therapy (IMRT) has gained popularity for CEC [3-5], and provides excellent dose coverage and conformity to the target volume compared to that by 3DCRT [6]. Helical tomotherapy (HT) can achieve higher degrees of dose conformity and homogeneity in the target compared to the traditional IMRT method, owing to a larger number of degrees of freedom in its beam arrangement using a rotating linear accelerator [7-9]. However, our previous study reported that using HT for CEC increases low-dose radiation in the lung compared to that by 3DCRT due to lateral directional beamlets that traverse both sides of the lung during gantry rotations [10]. Although the rate of radiation pneumonitis (RP) in CEC has not been determined, it is a common adverse event in thoracic oesophageal cancer, and low-dose irradiated lung volumes such as V5 and V10 have been reported as important predictive factors for RP [11-13]. An HT function termed "block plan" can restrict beamlets by using a virtual block during computation of dose optimization and may aid in dose reduction. Although only one phantom study has reported this planning technique supposing middle thoracic oesophageal cancer [14], there have been no reports regarding CEC, and the efficiency of this block plan in a clinical setting remains unclear.

Herein, to examine the lung-dose reducing efficacy of the block plan, we compared dosimetric parameters between non-block and block plans with modified conditions.

\section{Methods}

\section{Patients and simulation}

We retrospectively reviewed 20 patients diagnosed with CEC and treated with IMRT between March 2011 and March 2016 at the Aichi Cancer Center Hospital. Five patients were excluded due to exclusion of the lungs in the treatment-planning computed tomography $(\mathrm{CT})(N=2)$ and due to the target volume existing in the superior mandible $(N=2)$ or inferior tracheal bifurcation $(N=1)$ related to the involved lymph node. The remaining 15 patients were included and staged according to the 7th edition of the Union for International Cancer Control [15]. Before acquiring a planning CT scan, upper gastrointestinal endoscopy was performed with iodine staining, and, if necessary, a clip was placed to clarify the tumour extent, especially for superficial lesions. Patients were immobilized in the supine position using a thermoplastic mask which covered from the parietal to the shoulders, and the CT image was obtained with a 2-mm slice thickness. Existing contouring, such as target volumes and organs at risk (OARs), were checked and corrected, and virtual blocks were added as per the protocols described below.

\section{Virtual block}

Two types of virtual blocks were examined: a fanshaped block and a semi-circular block, contoured using the MIM Maestro ${ }^{\text {TM }}$ software (MIM Software, Inc., Cleveland, $\mathrm{OH}$ ). Firstly, we defined a perpendicular line passing through the tracheal bifurcation as a reference line (Fig. 1a, solid white line). In order to make the fanshaped block, the tracheal bifurcation was set as a central point (Fig. 1a, black point). For example, for block 40, two lines were drawn to make a fan-shaped block on the right side, with a central angle of 40 degrees (110 degrees and 70 degrees counter-clockwise from the reference line [Fig. 1a, dotted white-lines]). We contoured the right side of the lung between these lines and contoured the left side similarly. For a semicircular block, the distance from the tracheal bifurcation was measured. For example, for block 5, a 5-cm distance was measured (Fig. 1b, white arrow) and the right lung was contoured outside that region (Fig. 1b, white dot-area); the left side was contoured similarly. We made 5 fan-shaped blocks in increments of 10 degrees between 40 and 80 degrees (Fig. 1a), and delineated 4 semi-circular blocks in increments of $1 \mathrm{~cm}$ between 5 and $8 \mathrm{~cm}$ (Fig. 1b). However, a portion of the block within $1 \mathrm{~cm}$ of the planning target volume (PTV) was removed in order to avoid beam interference.

\section{Target and organs at risk setting}

The gross tumour volume of the primary lesion (GTVprimary) was defined by endoscopy and diagnostic imaging (barium contrast study, CT, or positron emission tomography-CT). The clinical target volume of the primary lesion (CTVprimary) was defined as the GTVprimary with $2 \mathrm{~cm}$ of cranio-caudal margin on the oesophageal wall and a $0.5-\mathrm{cm}$ margin in the lateral direction. The CTV of the nodal lesion (CTVnode) was defined as the involved lymph node, with a $0.5-\mathrm{cm}$ margin in every direction. The CTV for the prophylactic area (CTVprophylactic) ranged from the level III cervical node to the upper mediastinal node, including 101-107 lymph nodes (Japanese Classification of Esophageal Cancer $11^{\text {th }}$ edition) [16]. The PTV (PTVprimary/node/prophylactic) was defined as CTVprimary/node/prophylactic with a 0.5 - 


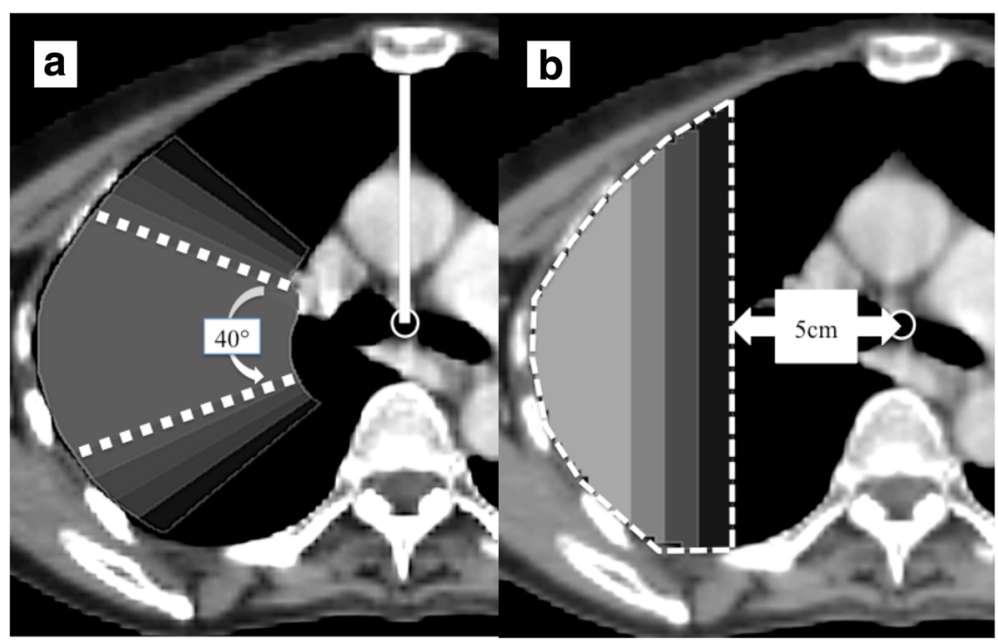

Fig. 1 Axial image of virtual blocks. a Five fan-shaped blocks in increments of 10 degrees between 40 and 80 degrees, which set the tracheal bifurcation as a central point. $\mathbf{b}$ Four semi-circular blocks in increments of $1 \mathrm{~cm}$ between 5 and $8 \mathrm{~cm}$ from the tracheal bifurcation

$\mathrm{cm}$ margin in all directions. The OARs included the lung, heart, thyroid, and spinal cord. For the spinal cord, a planning risk volume (PRV) with a 5-mm margin was created.

\section{Treatment system and planning optimization}

A Helical Tomotherapy Hi-Art treatment system (Accuray, Madison, WI) was used for delivering IMRT treatments. Planning Stations (Accuray, Madison, WI) were used to optimize the IMRT dose prescription. The planning parameter values were as follows: modulation factor: 2.2, pitch: 0.43 , and field width: $2.5 \mathrm{~cm}$ for all cases [17]. The superposition algorithm and a calculation matrix of $2 \mathrm{~mm}$ for all directions were used. Virtual blocks were used in two modes: complete mode, which restricts beamlets that pass the block, and directional mode, which permits beamlets that reach the block after passing through the PTVs. For the PTVprimary and PTVnode, $60 \mathrm{~Gy}$ in 30 fractions were delivered, while 48 Gy in 30 fractions were delivered for the PTVprophylactic, using a simultaneous integrated boost technique. The goal was to cover 95\% of each PTV with the prescribed dose (D95\%). Specifically, dose constraints were defined as follows: D98\% > 54.0 Gy (90\%), D95\% > 58 . 8 Gy (98\%), D50\% < 64.2 Gy (107\%), D10\% < 69.0 Gy (115\%), and D2\% < 72 Gy (120\%) for the PTVprimary and PTVnode; D98\% > 43.8 Gy (73\%), D95\% > 46.8 Gy (78\%), D50\% < 55.8 Gy (93\%), and D2\% <64.2 Gy (107\%) for the PTVprophylactic; Dmax < 52 Gy, D2\% $<50$ Gy for the PRV of the spinal cord; and Dmax $<75$ Gy (125\%) for the whole body. Nineteen plans (a non-block plan and block plans using the abovementioned two modes for 5 fan-shaped and 4 semicircular blocks) per patient were designed, and a total of 285 plans were compared. The same radiation oncologist that routinely makes the inverse planning performed all planning optimizations to prevent technical biases. The plans that did not meet the constraints were excluded from the analysis.

\section{Scoring and additional analyses}

Each block plan was scored to evaluate its efficacy. Based on previous studies on RP, the compliance criteria of the lung volume receiving 5 Gy (V5), 10 Gy (V10), $20 \mathrm{~Gy}$ (V20), and the absolute volume of the lung spared from 5 Gy (VS5) were defined as $55 \%, 37 \%, 25 \%$, and $1500 \mathrm{~mL}$, respectively $[11,12,18]$. Each plan was scored $(0-5)$ according to the grade of reduction in the lung dose (Table 1). The number of cases with plan approval (0-15) was added to this score to obtain a total block score (0-35). Three high-scoring blockage modes with scores $>30$ were analysed by one-way analysis of variance. The indices were calculated as follows:

$$
\begin{aligned}
\cdot \text { conformity index }(\mathrm{CI}) & =\text { Paddick CI } \\
& =(\text { TVPIV }) 2 /(\mathrm{TV} \times \mathrm{PIV}),
\end{aligned}
$$

[19].

where TVPIV: PTVprimary/node volume covered by the prescription isodose.

TV: PTVprimary/node volume.

PIV: prescription isodose

- homogeneity index $(\mathrm{HI})=(\mathrm{D} 2 \%-\mathrm{D} 98 \%) / \mathrm{D} 50 \%$,

[20].

where D2\%, D98\%, and D50\%: minimum doses in 2\%, $98 \%$, and $50 \%$ of the PTVprimary/node volume, respectively. 
Table 1 Definition of the scores

\begin{tabular}{|c|c|c|c|c|c|}
\hline & clear the criteria & reduce the criteria $20 \%$ & reduce the criteria $30 \%$ & reduce the criteria $40 \%$ & reduce the criteria $50 \%$ \\
\hline Score & 1 & 2 & 3 & 4 & 5 \\
\hline V5 (\%) & $44<L V \leqq 55$ & $38.5<\mathrm{LV} \leqq 44$ & $33<\mathrm{LV} \leqq 38.5$ & $27.5<\mathrm{LV} \leqq 33$ & $\mathrm{LV} \leqq 27.5$ \\
\hline V10 (\%) & $29.6<\mathrm{LV} \leqq 37$ & $25.9<\mathrm{LV} \leqq 29.6$ & $22.2<\mathrm{LV} \leqq 25.9$ & $18.5<\mathrm{LV} \leqq 22.2$ & $\mathrm{LV} \leqq 18.5$ \\
\hline V20 (\%) & $20<\mathrm{LV} \leqq 25$ & $17.5<\mathrm{LV} \leqq 20$ & $15<\mathrm{LV} \leqq 17.5$ & $12.5<\mathrm{LV} \leqq 15$ & $\mathrm{LV} \leqq 12.5$ \\
\hline VS5 (mL) & $1800>$ LV $\geqq 1500$ & $1950>L V \geqq 1800$ & $2100>$ LV $\geqq 1950$ & $2250>\mathrm{LV} \geqq 2100$ & $L V \geqq 2250$ \\
\hline
\end{tabular}

V5 lung volume receiving $5 \mathrm{~Gy}$, V10 lung volume receiving $10 \mathrm{~Gy}$, V20 lung volume receiving $20 \mathrm{~Gy}$, VS5 normal pulmonary volume of less than 5 Gy, $L V$ average lung volume

Delivery time was defined as the beam-on-time displayed on Planning Stations after the final calculation.

Statistical analysis was performed with EZR version 1.33 (Saitama Medical Center, Jichi Medical University, Saitama, Japan), which is based on R and R commander [21].

\section{Results}

\section{Patient characteristics}

The patient characteristics are listed in Table 2. Since elderly people and women were included, there were several patients with a low lung volume: the median volume was $3389 \mathrm{~mL}$ (range: 2319-4448 $\mathrm{mL}$ ). The primary tumours tended to be advance (T3-4), and 2 cases showed thyroidal invasion. The patients showed 1-6 lymph nodal invasions between the supraclavicular area and the tracheal bifurcation, except in 1 case.

\section{Presentation of a sample case}

Two dose distributions of a sample case are shown in Fig. 2. In the non-block plan, low-dose radiation spread widely in the lung (Fig. 2a). When we used the appropriate block, the dose gradient was very steep, and the lung dose was reduced (Fig. 2b). Dose-volume histograms for the lung and PTV primary/node are shown in Fig. 3. There was no significant difference in V20 of the lungs (approximately 15\% variation) between the plans; however, V5 or V10 was high in the non-block plan. In the block plan, we could reduce low-dose radiation in the lung while maintaining dose coverage and homogeneity to the PTVprimary/node.

\section{Lung dose and score for each block plan}

The mean values of the lung dose and score for each fan-shaped block are listed in Table 3. In the non-block plan group (controls), the plans satisfied the dose constraints in all 15 cases, with a score of 15. The V5 score, however, was 1 , since V5 fell below the compliance criteria in this group. In addition, this group was scored as 0,3 , and 1 for V10, V20, and VS5, respectively. Thus, the non-block plan group scored 20 in total. As none of the fan-shaped blocks in the complete mode plan were defined as approved plans, they could not be scored. In the directional mode, when using a block with a central angle of 50 or 60 degrees, the compliance rate of the restriction and the reduction in lung dose were the most well-balanced. When the central angle was 70 degrees or more, the constraints could not be met. The highest score of a fan-shaped block plan was 29. In plans using

Table 2 Patient characteristics

\begin{tabular}{|c|c|}
\hline No. of patients & 15 \\
\hline \multicolumn{2}{|l|}{ Age (years) } \\
\hline median & 64 \\
\hline range & $54-81$ \\
\hline \multicolumn{2}{|l|}{ Sex } \\
\hline Male & 10 \\
\hline Female & 5 \\
\hline \multicolumn{2}{|c|}{ Tumour size $(\mathrm{cm})$} \\
\hline median & 4 \\
\hline range & $2-10$ \\
\hline \multicolumn{2}{|l|}{$\mathrm{T}$ - category } \\
\hline 1 & 0 \\
\hline 2 & 3 \\
\hline 3 & 9 \\
\hline 4 & 3 \\
\hline \multicolumn{2}{|l|}{ N - category } \\
\hline 0 & 1 \\
\hline 1 & 7 \\
\hline 2 & 7 \\
\hline \multicolumn{2}{|l|}{ M - category } \\
\hline 0 & 15 \\
\hline 1 & 0 \\
\hline \multicolumn{2}{|l|}{ UICC stage } \\
\hline$\| \mathrm{A}$ & 1 \\
\hline$\| \mathrm{B}$ & 2 \\
\hline$\| \mathrm{A}$ & 6 \\
\hline$\| \mathrm{II}$ & 3 \\
\hline$\| I I C$ & 3 \\
\hline \multicolumn{2}{|c|}{ Lung volume (mL) } \\
\hline median & 3389 \\
\hline range & $2319-4448$ \\
\hline
\end{tabular}



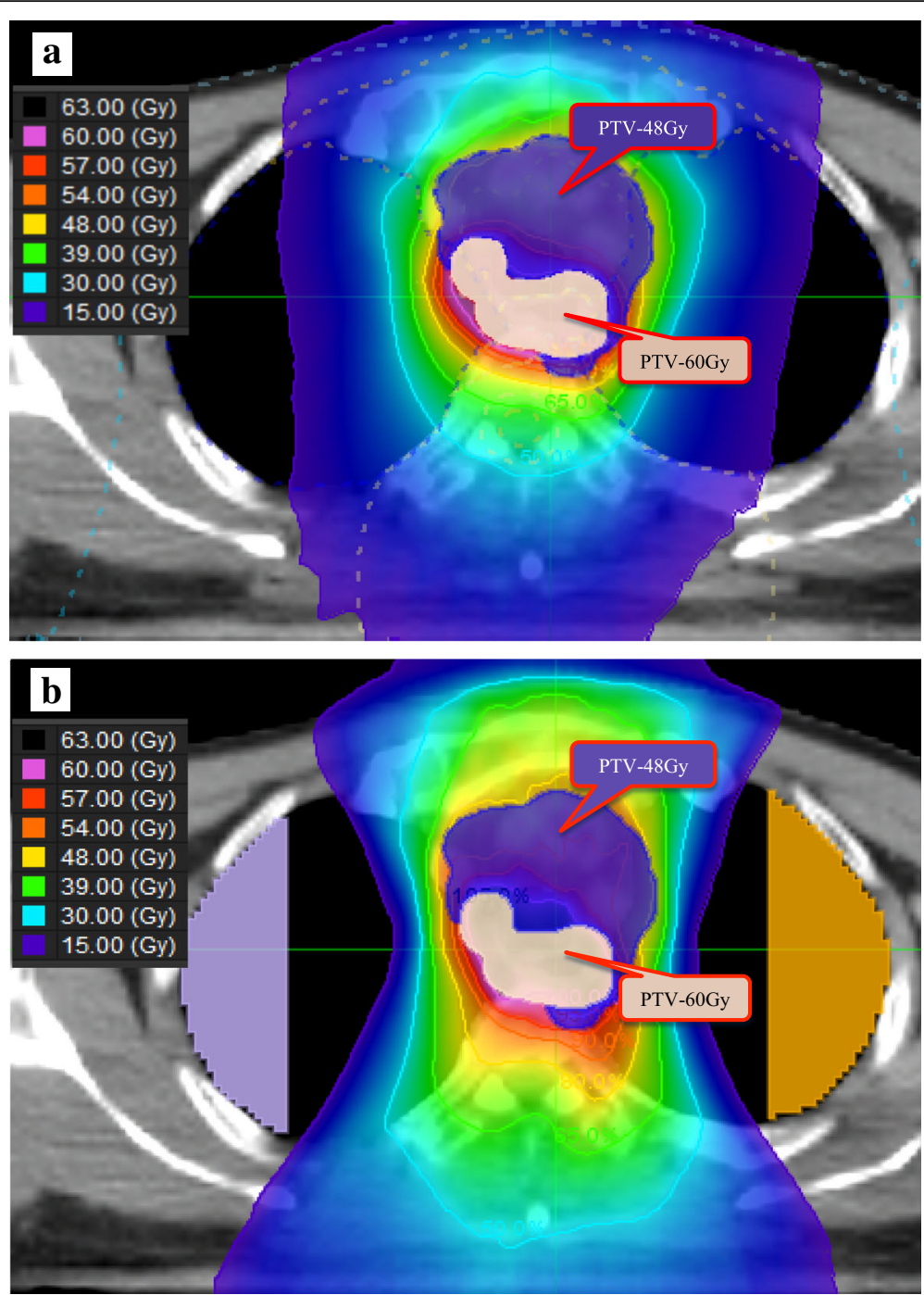

Fig. 2 Comparison of the dose distribution of non-block and block planning. a Non-block plan; (b) semi-circular plan for block 7. Each plan was made using the same target volume pertaining to a single patient. The dose gradient of block 7 was steeper than that of the non-block plan. Block 7: semi-circular virtual block that contoured the lungs outside a distance of $7 \mathrm{~cm}$ from the tracheal bifurcation

semi-circular blocks (Table 3), the blocks placed at a distance of $\geq 7 \mathrm{~cm}$ from the tracheal bifurcation were preferred. The highest score was 32, for a block 7 directional mode plan, followed by a score of 31 for directional mode and complete mode block 8 plans.

\section{Dosimetric parameter comparison among selected blocks}

We further analysed plans with scores $>30$. The plans using directional mode block 7 and block 8 were selected and compared with the non-block plan of the control group. As Table 4 shows, the lung dose was significantly reduced in all block plans. Without the block, there were 3,10 , and 5 cases (among 15), in which the lung volume exceeded the compliance criteria for V5, V10, and VS5, respectively. Alternatively, by using block 7 or 8 , we were able to meet the criteria in all cases.
Between the plan using the directional mode for block 8 and the non-block plan, there were no differences in the $\mathrm{CI}, \mathrm{HI}$, and body max dose, while compared to the other block plans, the CI of these plans decreased, and the $\mathrm{HI}$ and body max dose increased. By using a block, the maximum dose of the spinal cord PRV increased significantly but slightly: 47.9 to 49.4 Gy when using a block 8 in the directional mode. There was no difference in the average doses to the heart and thyroid among these plans. Although the dose delivery time was prolonged in all block plans, the increase in time was within $1 \mathrm{~min}$ for block 8 with directional mode.

\section{Discussion}

In recent years, IMRT has been used for oesophageal cancer [22, 23]. The fixed-field IMRT technique is more 


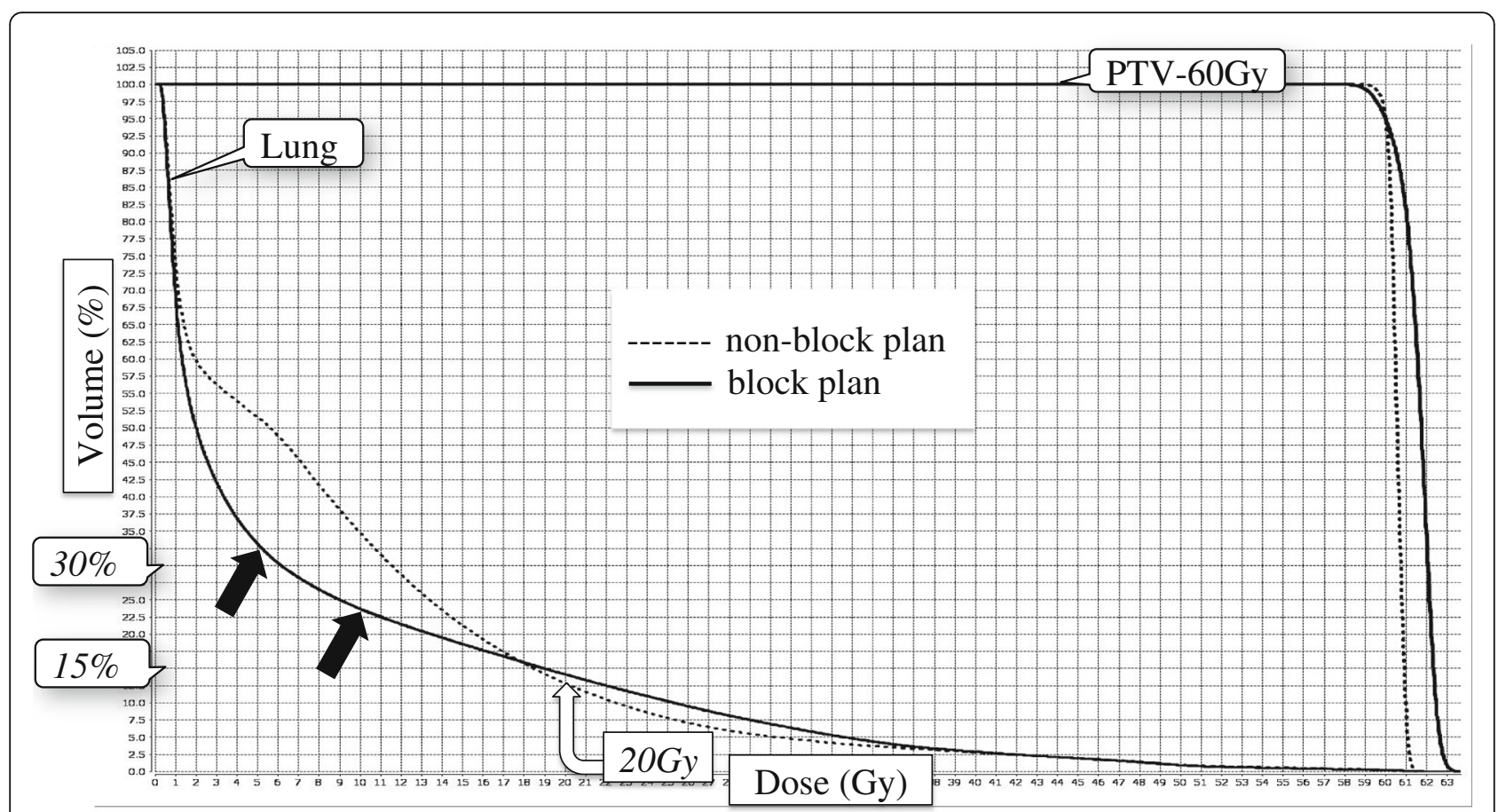

Fig. 3 Comparison of the dose-volume histogram of non-block and block planning. Although the V20 values of the lungs were comparable, with approximately $15 \%$ between the plans, V5 and V10 of the block plans were much lower than those in the non-block plan (black arrow).V20, the lung volume receiving $20 \mathrm{~Gy}$; V5, the lung volume receiving 5 Gy; V10, the lung volume receiving 10 Gy

suitable for thoracic oesophageal cancer rather than HT, considering the increase in lung and heart doses. Martin et al. [24] reported that the lung dose for the fixed-field IMRT with conformal arc technique was significantly reduced compared to HT for middle/distal oesophageal cancer, with acceptable PTV coverage; the mean V10 (66.2\%), V15 (34.8\%), and mean lung dose (MLD) (26. $3 \%$ ) of HT were improved to $40.3 \%, 25.2 \%$, and $21.2 \%$, respectively. As for $\mathrm{CEC}$, there are several reports that HT or volumetric-modulated arc therapy (VMAT) are preferable than fixed-field IMRT [8, 25-27]. Yin et al. [25] compared VMAT plans with conventional IMRT plans for 5 patients with CEC. According to their report, the CI was significantly higher in VMAT (single arc: 0 . 78, double arc: 0.8$)$ than in conventional $\operatorname{IMRT}(5,7$, and 9 fields: $0.62,0.66,0.73$, respectively), and the lung V30 was lower in VMAT (single arc: 12.52, double arc: 12.29) than in conventional IMRT (7 and 9 fields: 14.35 and 14.81, respectively). The difference in the preferred irradiation method between thoracic oesophageal cancer and CEC might be based on the difference in the range of the elective nodal irradiation area. Hirano et al. [28] reported that the incidences of cervical lymph nodal and upper mediastinal metastasis in 21 patients with CEC were $85.7 \%$ (especially wherein $43 \%$ were levels II-III) and $33.3 \%$, respectively, and asserted that neck lymph node (levels II-IV) and upper mediastinal dissection was crucial to improve the cure rate. Accordingly, many institutions have set the prophylactic area covered from level II or III of the cervical nodes to the subcarinal nodes of the mediastinum [5, 29], and the extensive exposure field where the body thickness differs thus causes difficulties in planning for CEC. Although HT could provide excellent target volume coverage and conformity for CEC, the pulmonary dose increment is a problem, and we therefore examined a novel planning method in this study. The block plan is different from the standard plan in the optimization process during the treatment planning only. The standard plan uses all beamlets in the optimization process. In contrast, the block plan excludes all or some of beamlets passing through the block. As a result, the block plan can provide a protective effect for normal tissue by multi-leaf collimators, effectively.

We aimed to establish a universal planning method, considering 3 points. Firstly, virtual blocks based on the tracheal bifurcation (which can be replicated easily) were defined. Secondly, 15 patients of both sexes and various ages, with varied tumour sizes and lung volumes, were included (Table 2), and the targets and OARs were described by a unified protocol. Finally, a total of 285 block plans were compared and the type, size, and mode of the final block plan were optimized. As a result, we were able to define an optimal block plan with a standardized study design (most well-balanced: block 8 directional mode plan), which was a significant achievement. 
Table 3 Comparison between plans using a fan-shaped block and those using a semi-circular block

\begin{tabular}{|c|c|c|c|c|c|c|c|c|}
\hline & & \multirow{2}{*}{$\begin{array}{l}\text { Block } \\
\text { mode }\end{array}$} & \multirow{2}{*}{$\begin{array}{l}\text { Number of } \\
\text { approved plan }\end{array}$} & \multirow[b]{2}{*}{ V5 (\%) } & \multicolumn{3}{|l|}{ Lung (mean \pm SD) } & \multirow[t]{2}{*}{ Score } \\
\hline & & & & & V10 (\%) & V20 (\%) & VS5 (mL) & \\
\hline Non-block & & & $15 / 15$ & $48.0 \pm 7.9$ & $39.4 \pm 6.9$ & $16.0 \pm 3.0$ & $1747 \pm 522$ & 20 \\
\hline \multirow[t]{10}{*}{ fan-shaped block } & Block 40 & C & $0 / 15$ & NA & NA & NA & NA & 0 \\
\hline & & D & $15 / 15$ & $38.7 \pm 6.4$ & $27.0 \pm 4.8$ & $12.9 \pm 2.9$ & $2049 \pm 527$ & 26 \\
\hline & Block 50 & C & $0 / 15$ & NA & NA & NA & NA & 0 \\
\hline & & D & $15 / 15$ & $36.5 \pm 5.9$ & $25.4 \pm 4.4$ & $12.6 \pm 2.7$ & $2124 \pm 535$ & 29 \\
\hline & Block 60 & C & $0 / 15$ & NA & NA & NA & NA & 0 \\
\hline & & D & $15 / 15$ & $34.4 \pm 5.7$ & $23.9 \pm 4.3$ & $13.0 \pm 3.3$ & $2197 \pm 560$ & 29 \\
\hline & Block 70 & C & $0 / 15$ & NA & NA & NA & NA & 0 \\
\hline & & D & $11 / 15$ & $33.5 \pm 5.9$ & $23.1 \pm 4.4$ & $12.3 \pm 2.8$ & $2223 \pm 585$ & 26 \\
\hline & Block 80 & C & $0 / 15$ & NA & NA & NA & NA & 0 \\
\hline & & $D$ & $3 / 15$ & $36.5 \pm 6.4$ & $25.5 \pm 4.9$ & $14.1 \pm 4.1$ & $2052 \pm 291$ & 16 \\
\hline \multirow[t]{8}{*}{ semi-circular block } & Block 5 & C & $0 / 15$ & NA & NA & NA & NA & 0 \\
\hline & & $D$ & $1 / 15$ & 29.7 & 20.3 & 12.4 & 2434 & 19 \\
\hline & Block 6 & $C$ & $0 / 15$ & NA & NA & NA & NA & 0 \\
\hline & & $D$ & $6 / 15$ & $29.1 \pm 3.3$ & $19.7 \pm 3.2$ & $12.0 \pm 3.1$ & $2396 \pm 288$ & 24 \\
\hline & Block 7 & $C$ & $6 / 15$ & $22.0 \pm 3.0$ & $17.0 \pm 2.3$ & $11.4 \pm 1.6$ & $2391 \pm 506$ & 26 \\
\hline & & $D$ & $14 / 15$ & $29.2 \pm 3.9$ & $20.6 \pm 3.5$ & $12.1 \pm 2.7$ & $2308 \pm 525$ & 32 \\
\hline & Block 8 & $C$ & $13 / 15$ & $26.4 \pm 4.4$ & $20.6 \pm 3.6$ & $13.3 \pm 2.9$ & $2454 \pm 586$ & 31 \\
\hline & & $D$ & $15 / 15$ & $31.3 \pm 4.8$ & $22.4 \pm 3.9$ & $13.2 \pm 3.0$ & $2299 \pm 574$ & 31 \\
\hline
\end{tabular}

V5 lung volume receiving $5 \mathrm{~Gy}$, V10 lung volume receiving $10 \mathrm{~Gy}$, V20 lung volume receiving $20 \mathrm{~Gy}$, VS5 normal pulmonary volume of less than 5 Gy, C complete block mode, $D$ directional block mode, $N A$ not available

In comparing the efficacy between the virtual blocks objectively, each block plan was scored.

In the non-block plan group, all 15 cases satisfied the dose constraints, with good CI (0.85) and HI (0.04), owing to the unconstrained irradiation beam angle. However, low-dose radiation spread widely in the lung, lowering the score (20 out of 35), and the mean V10 value was unable to clear the compliance criteria. Although we delineated the lung as an OAR and imposed strong restrictions, the effect of the lateral directional beamlets, which traverse both sides of the lung, was not suppressed. The fan-shaped block used in the directional

Table 4 Dosimetric parameter comparison among a selected series of blockage modes using a semi-circular block

\begin{tabular}{|c|c|c|c|c|c|c|c|c|}
\hline & & Non-block (control group) & Block 7 (D) & $p$ & Block 8 (C) & $p$ & Block 8 (D) & $p$ \\
\hline \multirow[t]{5}{*}{ Lung } & V5 (\%) & 48.0 & 29.2 & $<0.001$ & 26.4 & $<0.001$ & 31.3 & $<0.001$ \\
\hline & V10 (\%) & 39.4 & 20.6 & $<0.001$ & 20.6 & $<0.001$ & 22.4 & $<0.001$ \\
\hline & V20 (\%) & 16.0 & 12.1 & 0.002 & 13.3 & 0.046 & 13.2 & 0.028 \\
\hline & VS5 (mL) & 1747 & 2308 & 0.023 & 2454 & 0.004 & 2299 & 0.023 \\
\hline & mean dose (Gy) & 9.6 & 6.8 & $<0.001$ & 7.0 & $<0.001$ & 7.1 & $<0.001$ \\
\hline \multicolumn{2}{|c|}{ Conformity Index } & 0.85 & 0.77 & 0.012 & 0.79 & 0.131 & 0.82 & 0.616 \\
\hline \multicolumn{2}{|c|}{ Homogeneity Index } & 0.04 & 0.07 & 0.001 & 0.07 & 0.002 & 0.05 & 0.100 \\
\hline \multicolumn{2}{|c|}{ Spinal cord PRV max dose (Gy) } & 47.9 & 49.7 & $<0.001$ & 50.2 & $<0.001$ & 49.4 & 0.002 \\
\hline \multicolumn{2}{|c|}{ Body max dose (Gy) } & 63.3 & 64.7 & 0.005 & 65.2 & $<0.001$ & 63.9 & 0.317 \\
\hline \multicolumn{2}{|c|}{ Heart mean dose (Gy) } & 6.2 & 7.4 & 0.564 & 7.2 & 0.719 & 7.0 & 0.811 \\
\hline \multicolumn{2}{|c|}{ Thyroid mean dose (Gy) } & 53.6 & 54.0 & 0.964 & 55.4 & 0.315 & 54.0 & 0.968 \\
\hline \multicolumn{2}{|c|}{ Delivery time (min) } & 4.9 & 6.0 & $<0.001$ & 6.2 & $<0.001$ & 5.6 & 0.010 \\
\hline
\end{tabular}

V5 lung volume receiving $5 \mathrm{~Gy}$, V10 lung volume receiving $10 \mathrm{~Gy}$, V20 lung volume receiving 20 Gy, VS5 normal pulmonary volume of less than 5 Gy, $C$ complete block mode, $D$ directional block mode, $P R V$ planning risk volume 
mode, on the other hand, led to improved scores (Table 3). Since the irradiation beamlets would be restricted to protect the fan-shaped area in the lungs, when the central angle was larger, a larger lung volume would be protected. However, when the central angle was 70 degrees or more, the dose constraints of the spinal cord PRV were not met, because beamlets were directed dorsoventrally. None of the plans with complete mode groups for a fan-shaped block were defined as approved plans. Although the block sections overlapping with the PTV plus a $1-\mathrm{cm}$ area were removed, we were not able to plan optimization of the supraclavicular prophylactic area adequately. Semi-circular blocks were more effective than fanshaped blocks in reducing the lung dose, and the dose reduction improved with block size. In cases with underlying diseases like interstitial pneumonitis, it might be preferable to use block 7 for directional mode or block 8 for complete mode, in order to reduce the lung dose as much as possible. However, evaluation of the plan quality in the context of all parameters is required; it is important that high doses are not prescribed out of the PTV for a patient, and that the dose in the PTV is homogeneous. By using a block, the maximum dose of the spinal cord PRV increased slightly but significantly: for example, from 47.9 to 49.4 Gy when using a block 8 in the directional mode. It must be recognized that the option of future re-irradiation may be limited due to this slight increase. Therefore, when using a block, a lower spine dose plan should be considered, if all other parameters being equal. Lowering the irradiation time is also important in reducing the patient burden. Although prolongation of the irradiation time is unavoidable due to beamlet restriction, this time should be shortened as much as possible. Therefore, we consider that the best plan was the directional mode plan for block 8 , which could significantly reduce the lung dose without adversely affecting other dosimetric parameters (e.g. CI and $\mathrm{HI}$ ), while increasing the delivery time by under $1 \mathrm{~min}$. The most important contribution of our study is the universal block planning method for CEC. At first, we considered that the block size may need patient-specific modification, but the block 8 plan for the directional mode showed favourable results in all cases, across a large range of lung volumes (2319$4448 \mathrm{~mL}$ ). As shown in Table 3, the reduction in the lung dose was inadequate or did not clear the dose constraints when an unsuitable block was used. Since changes in the size of the block or the mode of the plan would require re-optimization, applying an optimized plan without changes could shorten planning times remarkably.

Dosimetric parameters of lung V5, V10, and VS5, which were used as compliance criteria in our study, are important factors associated with RP. Tanabe et al. [12] studied 86 patients with locally advanced oesophageal cancer treated with definitive CRT (radiation dose: 50.4 or 59.4 Gy). Patients with Grade 0-1 RP showed significantly lower V5 and V10 values for the whole lung compared to those with Grade 2-5 RP. The proposed plan led to advantageous V5 $(<55 \%)$ and V10 (<37\%) values, and conferred PTV conformity. Tsujino et al. [18] reported that $14(11.4 \%)$ of 122 patients with locally advanced non-small-cell lung cancer treated with concurrent CRT developed RP greater than or equal to Grade 3, and revealed that VS5 $<1500 \mathrm{~cm}^{3}$ was a significant risk factor for RP. We consider that using a block plan and meeting their criteria might reduce the $\mathrm{RP}$ risk. On the other hand, the non-block plan also showed low V20 and MLD values (16.0\% and $9.6 \mathrm{~Gy}$, respectively), since we analysed typical CEC patients with the target's inferior border set to the tracheal bifurcation. Chang et al. [14] reported a phantom study supposing middle thoracic oesophageal cancer, and reported a substantial reduction in the lung dose using a fan-shaped complete block compared to a non-block design (reductions in V20, V15, V10, V5, and MLD of 6. $3-8.6 \%, 16-23 \%, 42-57 \%, 42-66 \%$, and $5.2 \mathrm{~Gy}-7.5 \mathrm{~Gy}$, respectively). Since thoracic oesophageal invasion or a skip lesion in the thoracic oesophagus is often observed in $C E C$, the target's inferior border to the middle or lower thoracic oesophagus is planned to be expanded. When performing such an extended irradiation, we consider that a block plan can further reduce the RP risk. In addition, we are now able to use a TomoDirect mode that allows delivery of radiation at pre-established discrete angles with a fixed gantry [30]. Although the efficacy of the TomoDirect mode for oesophageal cancer has not yet been determined, Murai et al. [31] reported its usefulness in lung cancer compared to the Tomohelical mode (lung V5: $30 \pm 3 \%$ vs. $43 \pm 3 \%$, $P=0.005 ; n=18$ ). Thus, combining a TomoDirect mode and a block plan may result in a higher quality plan.

The most important limitation of our study is the lack of verification. We confirm that the passing rate of gamma analysis ( $2 \mathrm{~mm} / 2 \%$, threshold $20 \%$ ) for the 6 block plans that were actually used in the clinical treatment by the ArcCHECK quality assurance phantom (Sun Nuclear, Melbourne, FL) was more than 90\%. However, where the study plans were not used in practice, verification was not performed. The HT achieves highdose conformity according to accurate motion of the multileaf collimator. Since beamlets are steeply restricted according to a gantry angle, the block plan might be largely affected by setup error compared with the nonblock plan. It is thus essential to clarify the relation between positioning error and dose coverage of the PTV in future studies. Another limitation concerns the 
comparison of block plans. The scoring system used was adapted for comparing a large number of plans rapidly and was not based on established evidence. In addition, although the block 8 directional mode plan reduced the lung dose significantly compared with non-block plans, it was not always statistically superior compared with other block plans. Although more superior block types than those examined here may exist, our technique might be an effective alternative option, especially in institutions where block plans are not used.

\section{Conclusions}

When helical irradiation is performed in the usual way for CEC, compliance criteria of the lung dose are often exceeded. To reduce the RP risk, a novel planning method which can be applied universally, is required. The block plan using HT can significantly reduce the lung dose, with acceptable increment of a spinal dose and a slightly prolonged irradiation time. Use of a semi-circular block at a distance of $8 \mathrm{~cm}$ from the bifurcation with directional mode is recommended based on our analysis.

\section{Abbreviations \\ 3DCRT: 3-dimensional conformal radiotherapy; CEC: Cervical oesophageal carcinoma; Cl: Conformity index; CRT: Chemoradiotherapy; CT: Computed tomography; CTV: Clinical target volume; GTV: Gross tumour volume; HI: Homogeneity index; HT: Helical tomotherapy; IMRT: Intensity-modulated radiation therapy; MLD: Mean lung dose; OAR: Organ at risk; PRV: Planning risk volume; PTV: Planning target volume; RP: Radiation pneumonitis; VMAT: Volumetric-modulated arc therapy}

\section{Acknowledgments}

Parts of the study results were presented at the $5^{\text {th }}$ Japan-Taiwan Radiation Oncology Symposium (Kobe, 2017).

\section{Funding}

This work did not receive any funding.

\section{Availability of data and materials}

The datasets used and/or analysed during the current study are available from the corresponding author on reasonable request.

\section{Authors' contributions}

$\mathrm{Ml}$ and $\mathrm{HS}$ conceived the study, performed all analyses, and drafted the manuscript, supported by TI and TK. TA, HT, NT, CM, YK, and DK were involved in the study design and contributed significantly to the editing of the manuscript. All authors read and approved the final manuscript.

\section{Ethics approval and consent to participate}

All procedures involving human participants were in accordance with the ethical standards of the institutional research committee, and with the 1964 Helsinki declaration and its later amendments or comparable ethical standards.

\section{Consent for publication}

All patients gave written informed consent.

\section{Competing interests}

The authors declare that they have no competing interests.

\section{Publisher's Note}

Springer Nature remains neutral with regard to jurisdictional claims in published maps and institutional affiliations.
Received: 27 December 2017 Accepted: 26 March 2018

Published online: 04 April 2018

\section{References}

1. Tachimori Y, Ozawa S, Numasaki H, Fujishiro M, Matsubara H, Oyama T, Shinoda M, Toh Y, Udagawa H, Uno T. Registration Committee for Esophageal Cancer of the Japan esophageal S: comprehensive registry of esophageal Cancer in Japan, 2009. Esophagus. 2016;13:110-37.

2. Zenda S, Kojima T, Kato K, Izumi S, Ozawa T, Kiyota N, Katada C, Tsushima T, Ito $Y$, Akimoto T, et al. Multicenter phase 2 study of Cisplatin and 5fluorouracil with concurrent radiation therapy as an organ preservation approach in patients with Squamous cell carcinoma of the cervical esophagus. Int J Radiat Oncol Biol Phys. 2016;96:976-84.

3. McDowell LJ, Huang SH, Xu W, Che J, RKS W, Brierley J, Kim J, Cummings B, Waldron J, Bayley A, et al. Effect of intensity modulated radiation therapy with concurrent chemotherapy on survival for patients with cervical esophageal carcinoma. Int J Radiat Oncol Biol Phys. 2017;98:186-95.

4. Cao CN, Luo JW, Gao L, Xu GZ, Yi JL, Huang XD, Wang K, Zhang SP, Qu Y, Li SY, et al. Intensity-modulated radiotherapy for cervical esophageal squamous cell carcinoma: clinical outcomes and patterns of failure. Eur Arch Otorhinolaryngol. 2016;273:741-7

5. Zhang P, Xi M, Zhao L, Qiu B, Liu H, Hu YH, Liu MZ. Clinical efficacy and failure pattern in patients with cervical esophageal cancer treated with definitive chemoradiotherapy. Radiother Oncol. 2015;116:257-61.

6. Fenkell L, Kaminsky I, Breen S, Huang S, Van Prooijen M, Ringash J. Dosimetric comparison of IMRT vs. 3D conformal radiotherapy in the treatment of cancer of the cervical esophagus. Radiother Oncol. 2008;89:287-91.

7. van Vulpen $M$, Field C, Raaijmakers $C P$, Parliament $M B$, Terhaard $C H$, MacKenzie MA, Scrimger R, Lagendijk JJ, Fallone BG. Comparing step-andshoot IMRT with dynamic helical tomotherapy IMRT plans for head-andneck cancer. Int J Radiat Oncol Biol Phys. 2005;62:1535-9.

8. Yin Y, Chen J, Xing L, Dong X, Liu T, Lu J, Yu J. Applications of IMAT in cervical esophageal cancer radiotherapy: a comparison with fixed-field IMRT in dosimetry and implementation. J Appl Clin Med Phys. 2011;12:3343.

9. Gao M, Li Q, Ning Z, Gu W, Huang J, Mu J, Pei H. Dosimetric comparison between step-shoot intensity-modulated radiotherapy and volumetricmodulated arc therapy for upper thoracic and cervical esophageal carcinoma. Med Dosim. 2016:41:131-5.

10. Ito M, Kodaira T, Tachibana H, Tomita N, Makita C, Koide $Y$, Kato D, Abe T, Muro K, Tajika M, et al. Clinical results of definitive chemoradiotherapy for cervical esophageal cancer: comparison of failure pattern and toxicities between intensity-modulated radiotherapy and 3-dimensional conformal radiotherapy. Head Neck. 2017;39:2406-15.

11. Shaikh T, Churilla TM, Monpara P, Scott WJ, Cohen SJ, Meyer JE. Risk of radiation pneumonitis in patients receiving taxane-based trimodality therapy for locally advanced esophageal cancer. Pract Radiat Oncol. 2016;6:388-94.

12. Tanabe S, Myojin M, Shimizu S, Fujino M, Takahashi H, Shirato H, Ito YM, Ishikawa M, Hosokawa M. Dose-volume analysis for respiratory toxicity in intrathoracic esophageal cancer patients treated with definitive chemoradiotherapy using extended fields. J Radiat Res. 2013;54:1085-94.

13. Nomura M, Kodaira T, Furutani K, Tachibana H, Tomita N, Goto Y. Predictive factors for radiation pneumonitis in oesophageal cancer patients treated with chemoradiotherapy without prophylactic nodal irradiation. Br J Radiol. 2012;85:813-8.

14. Chang CH, Mok GS, Shueng PW, Yeh HP, Shiau AC, Tien HJ, Lin CT, Wu TH. Fan-shaped complete block on helical tomotherapy for esophageal cancer: a phantom study. Biomed Res Int. 2015;2015:959504.

15. Sobin LH, Gospodarowicz MK, Wittekind C. TNM classification of malignant tumours. 7th ed. Wiley; 2009. https://www.ncbi.nlm.nih.gov/nlmcatalog/ 101511218.

16. Society JE. Japanese classification of esophageal Cancer, 11th edition: part I. Esophagus. 2017;14(1):1-36.

17. Binny D, Lancaster CM, Harris S, Sylvander SR. Effects of changing modulation and pitch parameters on tomotherapy delivery quality assurance plans. J Appl Clin Med Phys. 2015;16:5282.

18. Tsujino K, Hashimoto T, Shimada T, Yoden E, Fujii O, Ota Y, Satouchi M, Negoro S, Adachi S, Soejima T. Combined analysis of V20, VS5, pulmonary fibrosis score on baseline computed tomography, and patient age improves prediction of severe radiation pneumonitis after concurrent chemoradiotherapy for locally advanced non-small-cell lung cancer. J Thorac Oncol. 2014;9:983-90. 
19. Paddick I. A simple scoring ratio to index the conformity of radiosurgical treatment plans. Technical note. J Neurosurg. 2000;93(Suppl 3):219-22.

20. Das IJ, Andersen A, Chen ZJ, Dimofte A, Glatstein E, Hoisak J, Huang L, Langer MP, Lee C, Pacella M, et al. State of dose prescription and compliance to international standard (ICRU-83) in intensity modulated radiation therapy among academic institutions. Pract Radiat Oncol. 2017;7:e145-55.

21. Kanda Y. Investigation of the freely available easy-to-use software 'EZR' for medical statistics. Bone Marrow Transplant. 2013;48:452-8.

22. Roeder F, Nicolay NH, Nguyen T, Saleh-Ebrahimi L, Askoxylakis V, Bostel T, Zwicker F, Debus J, Timke C, Huber PE. Intensity modulated radiotherapy (IMRT) with concurrent chemotherapy as definitive treatment of locally advanced esophageal cancer. Radiat Oncol. 2014;9:191.

23. Lin SH, Wang L, Myles B, Thall PF, Hofstetter WL, Swisher SG, Ajani JA, Cox $J D$, Komaki R, Liao Z. Propensity score-based comparison of long-term outcomes with 3-dimensional conformal radiotherapy vs intensitymodulated radiotherapy for esophageal cancer. Int J Radiat Oncol Biol Phys. 2012:84:1078-85

24. Martin S, Chen JZ, Rashid Dar A, Yartsev S. Dosimetric comparison of helical tomotherapy, RapidArc, and a novel IMRT \& arc technique for esophageal carcinoma. Radiother Oncol. 2011;101:431-7.

25. Yin L, Wu H, Gong J, Geng JH, Jiang F, Shi AH, Yu R, Li YH, Han SK, Xu B, Zhu GY. Volumetric-modulated arc therapy vs. C-IMRT in esophageal cancer: a treatment planning comparison. World J Gastroenterol. 2012;18:5266-75.

26. Wang YC, Chen SW, Chien CR, Hsieh TC, Yu CY, Kuo YC, Yang SN, Kao CH, Liang JA. Radiotherapy for esophageal cancer using simultaneous integrated boost techniques: dosimetric comparison of helical TomoTherapy, volumetricmodulated arc therapy (RapidArc) and dynamic intensity-modulated radiotherapy. Technol Cancer Res Treat. 2013;12:485-91.

27. Lin JC, Tsai JT, Chang CC, Jen YM, Li MH, Liu WH. Comparing treatment plan in all locations of esophageal cancer: volumetric modulated arc therapy versus intensity-modulated radiotherapy. Medicine (Baltimore). 2015;94:e750.

28. Hirano S, Nagahara K, Moritani S, Kitamura M, Takagita S. Upper mediastinal node dissection for hypopharyngeal and cervical esophageal carcinomas. Ann Otol Rhinol Laryngol. 2007;116:290-6.

29. Yang H, Feng C, Cai BN, Yang J, Liu HX, Ma L. Comparison of threedimensional conformal radiation therapy, intensity-modulated radiation therapy, and volumetric-modulated arc therapy in the treatment of cervical esophageal carcinoma. Dis Esophagus. 2017;30(2):1-8.

30. Franco P, Catuzzo P, Cante D, La Porta MR, Sciacero P, Girelli G, Casanova Borca $V$, Pasquino M, Numico G, Tofani S, et al. TomoDirect: an efficient means to deliver radiation at static angles with tomotherapy. Tumori. 2011; 97:498-502.

31. Murai $T$, Shibamoto $Y$, Manabe $Y$, Murata $R$, Sugie $C$, Hayashi $A$, Ito $H$, Miyoshi $Y$. Intensity-modulated radiation therapy using static ports of tomotherapy (TomoDirect): comparison with the TomoHelical mode. Radiat Oncol. 2013;8:68

\section{Submit your next manuscript to BioMed Central and we will help you at every step:}

- We accept pre-submission inquiries

- Our selector tool helps you to find the most relevant journal

- We provide round the clock customer support

- Convenient online submission

- Thorough peer review

- Inclusion in PubMed and all major indexing services

- Maximum visibility for your research

Submit your manuscript at www.biomedcentral.com/submit 